\title{
Combining Recurrent and Convolutional Neural Networks for Relation Classification
}

\author{
Ngoc Thang Vu ${ }^{1,2}$ and Heike Adel ${ }^{1}$ and Pankaj Gupta ${ }^{3}$ and Hinrich Schütze ${ }^{1}$ \\ ${ }^{1}$ Center for Information and Language Processing, LMU Munich \\ Oettingenstr. 67, 80538 Munich, Germany \\ ${ }^{2}$ Institute for Natural Language Processing, University of Stuttgart \\ Pfaffenwaldring 5b, 70569 Stuttgart, Germany \\ ${ }^{3}$ Siemens Corporate Technology - Knowledge Modeling and Retrieval \\ Otto-Hahn-Ring 6, 81739 Munich, Germany \\ thang.vulims.uni-stuttgart.de I heike.adel@cis.lmu.de \\ gupta.pankaj.ext@siemens.com | inquiries@cislmu.org
}

\begin{abstract}
This paper investigates two different neural architectures for the task of relation classification: convolutional neural networks and recurrent neural networks. For both models, we demonstrate the effect of different architectural choices. We present a new context representation for convolutional neural networks for relation classification (extended middle context). Furthermore, we propose connectionist bi-directional recurrent neural networks and introduce ranking loss for their optimization. Finally, we show that combining convolutional and recurrent neural networks using a simple voting scheme is accurate enough to improve results. Our neural models achieve state-of-the-art results on the SemEval 2010 relation classification task.
\end{abstract}

\section{Introduction}

Relation classification is the task of assigning sentences with two marked entities to a predefined set of relations. The sentence "We poured the $<e 1>$ milk $</ e 1>$ into the $<e 2>$ pumpkin mixture $</$ e $2>$.", for example, expresses the relation Entity-Destination $(e 1, e 2)$. While early research mostly focused on support vector machines or maximum entropy classifiers (Rink and Harabagiu, 2010a; Tratz and Hovy, 2010), recent research showed performance improvements by applying neural networks (NNs) (Socher et al., 2012; Zeng et al., 2014; Yu et al., 2014; Nguyen and Grishman, 2015; Dos Santos et al., 2015; Zhang and Wang, 2015) on the benchmark data from SemEval 2010 shared task 8 (Hendrickx et al., 2010) .
This study investigates two different types of NNs: recurrent neural networks (RNNs) and convolutional neural networks (CNNs) as well as their combination. We make the following contributions:

(1) We propose extended middle context, a new context representation for $\mathrm{CNNs}$ for relation classification. The extended middle context uses all parts of the sentence (the relation arguments, left of the relation arguments, between the arguments, right of the arguments) and pays special attention to the middle part.

(2) We present connectionist bi-directional RNN models which are especially suited for sentence classification tasks since they combine all intermediate hidden layers for their final decision. Furthermore, the ranking loss function is introduced for the RNN model optimization which has not been investigated in the literature for relation classification before.

(3) Finally, we combine CNNs and RNNs using a simple voting scheme and achieve new state-of-theart results on the SemEval 2010 benchmark dataset.

\section{Related Work}

In 2010, manually annotated data for relation classification was released in the context of a SemEval shared task (Hendrickx et al., 2010). Shared task participants used, i.a., support vector machines or maximum entropy classifiers (Rink and Harabagiu, 2010a; Tratz and Hovy, 2010). Recently, their results on this data set were outperformed by applying NNs (Socher et al., 2012; Zeng et al., 2014; Yu et al., 2014; Nguyen and Grishman, 2015; Dos Santos et al., 2015). 
Zeng et al. (2014) built a CNN based only on the context between the relation arguments and extended it with several lexical features. Kim (2014) and others used convolutional filters of different sizes for CNNs. Nguyen and Grishman (2015) applied this to relation classification and obtained improvements over single filter sizes. Dos Santos et al. (2015) replaced the softmax layer of the CNN with a ranking layer. They showed improvements and published the best result so far on the SemEval dataset, to our knowledge.

Socher et al. (2012) used another NN architecture for relation classification: recursive neural networks that built recursive sentence representations based on syntactic parsing. In contrast, Zhang and Wang (2015) investigated a temporal structured RNN with only words as input. They used a bi-directional model with a pooling layer on top.

\section{Convolutional Neural Networks (CNN)}

CNNs perform a discrete convolution on an input matrix with a set of different filters. For NLP tasks, the input matrix represents a sentence: Each column of the matrix stores the word embedding of the corresponding word. By applying a filter with a width of, e.g., three columns, three neighboring words (trigram) are convolved. Afterwards, the results of the convolution are pooled. Following Collobert et al. (2011), we perform max-pooling which extracts the maximum value for each filter and, thus, the most informative n-gram for the following steps. Finally, the resulting values are concatenated and used for classifying the relation expressed in the sentence.

\subsection{Input: Extended Middle Context}

One of our contributions is a new input representation especially designed for relation classification. The contexts are split into three disjoint regions based on the two relation arguments: the left context, the middle context and the right context. Since in most cases the middle context contains the most relevant information for the relation, we want to focus on it but not ignore the other regions completely. Hence, we propose to use two contexts: (1) a combination of the left context, the left entity and the middle context; and (2) a combination of the middle context, the right entity and the right context.
Due to the repetition of the middle context, we force the network to pay special attention to it. The two contexts are processed by two independent convolutional and max-pooling layers. After pooling, the results are concatenated to form the sentence representation. Figure 1 depicts this procedure. It shows an examplary sentence: "He had chest pain and $<e 1>$ headaches $</ e 1>$ from $<e 2>$ mold $</ e 2>$ in the bedroom." If we only considered the middle context "from", the network might be tempted to predict a relation like Entity-Origin (e1,e2) . However, by also taking the left and right context into account, the model can detect the relation Cause-Effect $(e 2, e 1)$. While this could also be achieved by integrating the whole context into the model, using the whole context can have disadvantages for longer sentences: The max pooling step can easily choose a value from a part of the sentence which is far away from the mention of the relation. With splitting the context into two parts, we reduce this danger. Repeating the middle context increases the chance for the max pooling step to pick a value from the middle context.

\subsection{Convolutional Layer}

Following previous work (e.g., (Nguyen and Grishman, 2015), (Dos Santos et al., 2015)), we use 2D filters spanning all embedding dimensions. After convolution, a max pooling operation is applied that stores only the highest activation of each filter. We apply filters with different window sizes 2-5 (multiwindows) as in (Nguyen and Grishman, 2015), i.e. spanning a different number of input words.

\section{Recurrent Neural Networks (RNN)}

Traditional RNNs consist of an input vector, a history vector and an output vector. Based on the representation of the current input word and the previous history vector, a new history is computed. Then, an output is predicted (e.g., using a softmax layer). In contrast to most traditional RNN architectures, we use the RNN for sentence modeling, i.e., we predict an output vector only after processing the whole sentence and not after each word. Training is performed using backpropagation through time (Werbos, 1990) which unfolds the recurrent computations of the history vector for a certain number of time steps. To 


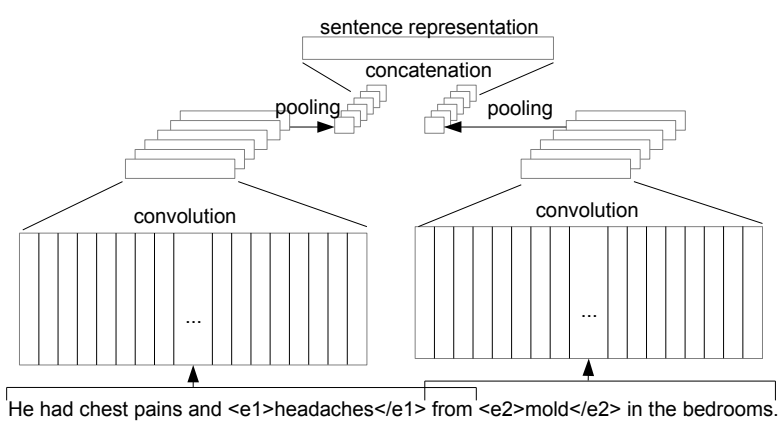

Figure 1: CNN with extended contexts

avoid exploding gradients, we use gradient clipping with a threshold of 10 (Pascanu et al., 2012).

\subsection{Input of the RNNs}

Initial experiments showed that using trigrams as input instead of single words led to superior results. Hence, at timestep $t$ we do not only give word $w_{t}$ to the model but the trigram $w_{t-1} w_{t} w_{t+1}$ by concatenating the corresponding word embeddings.

\subsection{Connectionist Bi-directional RNNs}

Especially for relation classification, the processing of the relation arguments might be easier with knowledge of the succeeding words. Therefore in bi-directional RNNs, not only a history vector of word $w_{t}$ is regarded but also a future vector. This leads to the following conditioned probability for the history $h_{t}$ at time step $t \in[1, n]$ :

$$
\begin{array}{r}
h_{f_{t}}=f\left(U_{f} \cdot w_{t}+V \cdot h_{f_{t-1}}\right) \\
h_{b_{t}}=f\left(U_{b} \cdot w_{n-t+1}+B \cdot h_{b_{t+1}}\right) \\
h_{t}=f\left(h_{b_{t}}+h_{f_{t}}+H \cdot h_{t-1}\right)
\end{array}
$$

Thus, the network can be split into three parts: a forward pass which processes the original sentence word by word (Equation 1); a backward pass which processes the reversed sentence word by word (Equation 2); and a combination of both (Equation 3). All three parts are trained jointly. This is also depicted in Figure 2.

Combining forward and backward pass by adding their hidden layer is similar to (Zhang and Wang, 2015). We, however, also add a connection to the previous combined hidden layer with weight $H$ to be able to include all intermediate hidden layers into the final decision of the network (see Equation 3). We call this "connectionist bi-directional RNN".

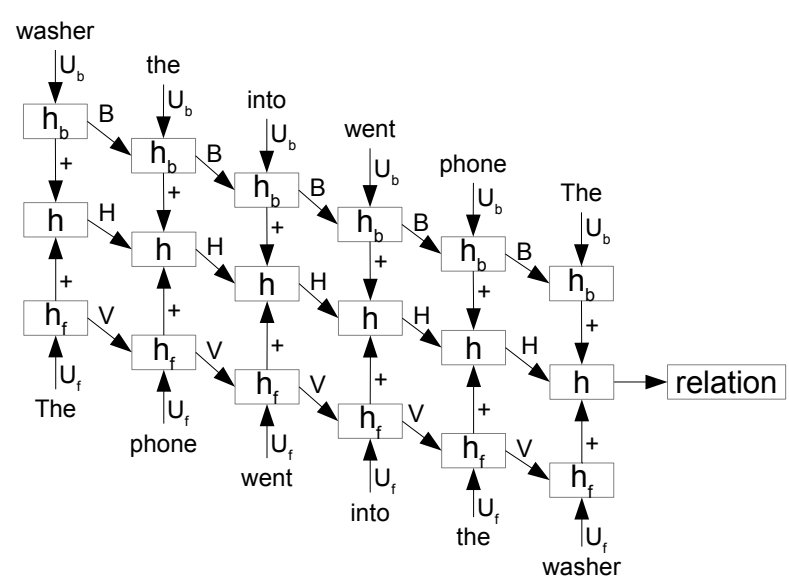

Figure 2: Connectionist bi-directional RNN

In our experiments, we compare this RNN with uni-directional RNNs and bi-directional RNNs without additional hidden layer connections.

\section{Model Training}

\subsection{Word Representations}

Words are represented by concatenated vectors: a word embedding and a position feature vector.

Pretrained word embeddings. In this study, we used the word2vec toolkit to train embeddings on an English Wikipedia from May 2014. We only considered words appearing more than 100 times and added a special PADDING token for convolution. This results in an embedding training text of about 485,000 terms and $6.7 \cdot 10^{9}$ tokens. During model training, the embeddings are updated.

Position features. We incorporate randomly initialized position embeddings similar to Zeng et al. (2014), Nguyen and Grishman (2015) and Dos Santos et al. (2015). In our RNN experiments, we investigate different possibilities of integrating position information: position embeddings, position embeddings with entity presence flags (flags indicating whether the current word is one of the relation arguments), and position indicators (Zhang and Wang, 2015).

\subsection{Objective Function: Ranking Loss}

Ranking. We applied the ranking loss function proposed in Dos Santos et al. (2015) to train our models. It maximizes the distance between the true label $y^{+}$ and the best competitive label $c^{-}$given a data point 
$x$. The objective function is

$$
\begin{aligned}
L & =\log \left(1+\exp \left(\gamma\left(m^{+}-s_{\theta}(x)_{y^{+}}\right)\right)\right) \\
& +\log \left(1+\exp \left(\gamma\left(m^{-}+s_{\theta}(x)_{c^{-}}\right)\right)\right)
\end{aligned}
$$

with $s_{\theta}(x)_{y^{+}}$and $s_{\theta}(x)_{c^{-}}$being the scores for the classes $y^{+}$and $c^{-}$respectively. The parameter $\gamma$ controls the penalization of the prediction errors and $\mathrm{m}^{+}$and $\mathrm{m}^{-}$are margins for the correct and incorrect classes. Following Dos Santos et al. (2015), we set $\gamma=2, m^{+}=2.5, m^{-}=0.5$. We do not learn a pattern for the class Other but increase its difference to the best competitive label by using only the second summand in Equation 4 during training.

\section{Experiments and Results}

We used the relation classification dataset of the SemEval 2010 task 8 (Hendrickx et al., 2010). It consists of sentences which have been manually labeled with 19 relations ( 9 directed relations and one artificial class Other). 8,000 sentences have been distributed as training set and 2,717 sentences served as test set. For evaluation, we applied the official scoring script and report the macro F1 score which also served as the official result of the shared task.

RNN and CNN models were implemented with theano (Bergstra et al., 2010; Bastien et al., 2012). For all our models, we use L2 regularization with a weight of 0.0001 . For CNN training, we use mini batches of 25 training examples while we perform stochastic gradient descent for the RNN. The initial learning rates are 0.2 for the $\mathrm{CNN}$ and 0.01 for the RNN. We train the models for $10(\mathrm{CNN})$ and 50 (RNN) epochs without early stopping. As activation function, we apply tanh for the CNN and capped ReLU for the RNN. For tuning the hyperparameters, we split the training data into two parts: $6.5 \mathrm{k}$ (training) and $1.5 \mathrm{k}$ (development) sentences. We also tuned the learning rate schedule on dev.

Beside of training single models, we also report ensemble results for which we combined the presented single models with a voting process.

\subsection{Performance of CNNs}

As a baseline system, we implemented a CNN similar to the one described by Zeng et al. (2014). It consists of a standard convolutional layer with filters with only one window size, followed by a softmax

\begin{tabular}{l|l} 
CNN & F1 \\
\hline Baseline (emb dim: 50) & 73.0 \\
+ position features & $78.6^{*}$ \\
+ multi-windows features map & 78.7 \\
+ ranking layer & $81.9^{*}$ \\
+ extended middle context & 82.2 \\
\hline + increase emb dim to 400 & $83.9^{*}$ \\
\hline ensemble & $\mathbf{8 4 . 2}$
\end{tabular}

Table 1: F1 score of $\mathrm{CNN}$ and its components, * indicates statisticial significance compared to the result in the line above (z-test, $p<0.05$ )

layer. As input it uses the middle context. In contrast to Zeng et al. (2014), our CNN does not have an additional fully connected hidden layer. Therefore, we increased the number of convolutional filters to 1200 to keep the number of parameters comparable. With this, we obtain a baseline result of 73.0. After including 5 dimensional position features, the performance was improved to 78.6 (comparable to 78.9 as reported by Zeng et al. (2014) without linguistic features).

In the next step, we investigate how this result changes if we successively add further features to our CNN: multi-windows for convolution (window sizes: 2,3,4,5 and 300 feature maps each), ranking layer instead of softmax and our proposed extended middle context. Table 1 shows the results. Note that all numbers are produced by CNNs with a comparable number of parameters. We also report F1 for increasing the word embedding dimensionality from 50 to 400 . The position embedding dimensionality is 5 in combination with 50 dimensional word embeddings and 35 with 400 dimensional word embeddings. Our results show that especially the ranking layer and the embedding size have an important impact on the performance.

\subsection{Performance of RNNs}

As a baseline for the RNN models, we apply a unidirectional RNN which predicts the relation after processing the whole sentence. With this model, we achieve an F1 score of 61.2 on the SemEval test set.

Afterwards, we investigate the impact of different position features on the performance of unidirectional RNNs (position embeddings, position embeddings concatenated with a flag indicating whether the current word is an entity or not, and 


\begin{tabular}{l|l} 
RNN & $\mathrm{F} 1$ \\
\hline uni-directional (Baseline, emb dim: 50) & 61.2 \\
uni-directional + position embs & $68.3^{*}$ \\
uni-directional + position embs + entity flag & $73.1^{*}$ \\
uni-directional + position indicators & 73.4 \\
\hline bi-directional + position indicators & $74.2^{*}$ \\
\hline connectionist-bi-directional+position indicators & $78.4^{*}$ \\
+ ranking layer & $81.4^{*}$ \\
+ increase emb dim to 400 & $82.5^{*}$ \\
\hline ensemble & $\mathbf{8 3 . 4}$
\end{tabular}

Table 2: $\mathrm{F} 1$ score of $\mathrm{RNN}$ and its components, * indicates statisticial significance compared to the result in the line above (z-test, $p<0.05$ )

position indicators (Zhang and Wang, 2015)). The results indicate that position indicators (i.e. artificial words that indicate the entity presence) perform the best on the SemEval data. We achieve an F1 score of 73.4 with them. However, the difference to using position embeddings with entity flags is not statistically significant.

Similar to our CNN experiments, we successively vary the RNN models by using bi-directionality, by adding connections between the hidden layers ("connectionist"), by applying ranking instead of softmax to predict the relation and by increasing the word embedding dimension to 400 .

The results in Table 2 show that all of these variations lead to statistically significant improvements. Especially the additional hidden layer connections and the integration of the ranking layer have a large impact on the performance.

\subsection{Combination of CNNs and RNNs}

Finally, we combine our CNN and RNN models using a voting process. For each sentence in the test set, we apply several CNN and RNN models presented in Tables 1 and 2 and predict the class with the most votes. In case of a tie, we pick one of the most frequent classes randomly. The combination achieves an F1 score of 84.9 which is better than the performance of the two NN types alone. It, thus, confirms our assumption that the networks provide complementary information: while the RNN computes a weighted combination of all words in the sentence, the CNN extracts the most informative ngrams for the relation and only considers their resulting activations.

\begin{tabular}{l|l} 
Classifier & F1 \\
\hline SVM (Rink and Harabagiu, 2010b) & 82.2 \\
\hline RNN (Socher et al., 2012) & 77.6 \\
MVRNN (Socher et al., 2012) & 82.4 \\
\hline CNN (Zeng et al., 2014) & 82.7 \\
\hline FCM (Yu et al., 2014) & 83.0 \\
\hline bi-RNN (Zhang and Wang, 2015) & 82.5 \\
\hline CR-CNN (Dos Santos et al., 2015) & 84.1 \\
\hline \hline R-RNN & 83.4 \\
ER-CNN & 84.2 \\
ER-CNN + R-RNN & $\mathbf{8 4 . 9}$
\end{tabular}

Table 3: State-of-the-art results for relation classification

\subsection{Comparison with State of the Art}

Table 3 shows the results of our models ER$\mathrm{CNN}$ (extended ranking CNN) and R-RNN (ranking $\mathrm{RNN}$ ) in the context of other state-of-the-art models. Our proposed models obtain state-of-the-art results on the SemEval 2010 task 8 data set without making use of any linguistic features.

\section{Conclusion}

In this paper, we investigated different features and architectural choices for convolutional and recurrent neural networks for relation classification without using any linguistic features. For convolutional neural networks, we presented a new context representation for relation classification. Furthermore, we introduced connectionist recurrent neural networks for sentence classification tasks and performed the first experiments with ranking recurrent neural networks. Finally, we showed that even a simple combination of convolutional and recurrent neural networks improved results. With our neural models, we achieved new state-of-the-art results on the SemEval 2010 task 8 benchmark data.

\section{Acknowledgments}

Heike Adel is a recipient of the Google European Doctoral Fellowship in Natural Language Processing and this research is supported by this fellowship.

This research was also supported by Deutsche Forschungsgemeinschaft: grant SCHU 2246/4-2.

\section{References}

Frédéric Bastien, Pascal Lamblin, Razvan Pascanu, James Bergstra, Ian J. Goodfellow, Arnaud Berg- 
eron, Nicolas Bouchard, and Yoshua Bengio. 2012. Theano: new features and speed improvements. Deep Learning and Unsupervised Feature Learning NIPS 2012 Workshop.

James Bergstra, Olivier Breuleux, Frédéric Bastien, Pascal Lamblin, Razvan Pascanu, Guillaume Desjardins, Joseph Turian, David Warde-Farley, and Yoshua Bengio. 2010. Theano: a CPU and GPU math expression compiler. In Proceedings of the Python for Scientific Computing Conference (SciPy).

Ronan Collobert, Jason Weston, Léon Bottou, Michael Karlen, Koray Kavukcuoglu, and Pavel Kuksa. 2011. Natural language processing (almost) from scratch. The Journal of Machine Learning Research, 12:24932537.

Cícero Nogueira Dos Santos, Bing Xiang, and Bowen Zhou. 2015. Classifying relations by ranking with convolutional neural networks. In Proceedings of $A C L$. Association for Computational Linguistics.

Iris Hendrickx, Su Nam Kim, Zornitsa Kozareva, Preslav Nakov, Diarmuid Ó Séaghdha, Sebastian Padó, Marco Pennacchiotti, Lorenza Romano, and Stan Szpakowicz. 2010. Semeval-2010 task 8: Multi-way classification of semantic relations between pairs of nominals. In Proceedings of the Workshop on SemEval. Association for Computational Linguistics.

Yoon Kim. 2014. Convolutional neural networks for sentence classification. In Proceedings of EMNLP. Association for Computational Linguistics.

Thien Huu Nguyen and Ralph Grishman. 2015. Relation extraction: Perspective from convolutional neural networks. In Proceedings of the NAACL Workshop on Vector Space Modeling for NLP. Association for Computational Linguistics.

Razvan Pascanu, Tomas Mikolov, and Yoshua Bengio. 2012. Understanding the exploding gradient problem. Computing Research Repository.

Bryan Rink and Sanda Harabagiu. 2010a. Utd: Classifying semantic relations by combining lexical and semantic resources. In Proceedings of the Workshop on SemEval. Association for Computational Linguistics.

Bryan Rink and Sanda Harabagiu. 2010b. Utd: Classifying semantic relations by combining lexical and semantic resources. In Proceedings of the Workshop on SemEval, pages 256-259. Association for Computational Linguistics.

Richard Socher, Brody Huval, Christopher D Manning, and Andrew Y Ng. 2012. Semantic compositionality through recursive matrix-vector spaces. In Proceedings of EMNLP / CoNLL. Association for Computational Linguistics.

Stephen Tratz and Eduard Hovy. 2010. Isi: automatic classification of relations between nominals using a maximum entropy classifier. In Proceedings of the Workshop on SemEval. Association for Computational Linguistics.

Paul J Werbos. 1990. Backpropagation through time: what it does and how to do it. Proceedings of the IEEE.

Mo Yu, Matthew Gormley, and Mark Dredze. 2014. Factor-based compositional embedding models. In Proceedings of the NIPS Workshop on Learning Semantics.

Daojian Zeng, Kang Liu, Siwei Lai, Guangyou Zhou, and Jun Zhao. 2014. Relation classification via convolutional deep neural network. In Proceedings of COLING.

Dongxu Zhang and Dong Wang. 2015. Relation classification via recurrent neural network. In ArXiv. 